\title{
Effect of age on inter and intra-subject variability in acceptable noise level (ANL) in listeners with normal hearing
}

\author{
Bankole K. Fasanya $^{a^{*}}$, Oluseun Omotoso ${ }^{b}$ and Olariyike A. Fasanya ${ }^{a}$
}

${ }^{a}$ North Carolina A \& T State University, Greensboro, NC27422

${ }^{b}$ Maryland department of the Environment 1800, Washington Blvd Baltimore MD 21230

CHRON I C L E

\section{Article history:}

Received September 28, 2012

Received in revised format

19 November 2012

Accepted 20 November 2012

Available online

November 272012

\section{Keywords:}

Noise Level

Background noise

Accurate hearing

\section{A B S T R A C T}

\begin{abstract}
Several industrial engineering and psychological studies have shown that noise affects the important aspects of communication for both adults and children. For speech understanding by hearing aids users and language development in children, an accurate hearing is very important. A metric has been developed for measuring an individual's acceptance of noise while listening to speech in quiet. This metric is known as Acceptable Noise Levels (ANLs). Studies have shown large inter-subject variability in acceptance of background noise. An argument has been made that an acceptance of background noise is a "means" that will help to solve the puzzle and monumental problem of hearing aid rejection. Meanwhile, within subject age dependency has not been investigated. This study is conducted to determine if ANL inter and intra-subject variability under music signal depends on age. Twenty subjects participated in the study (average age $=29 ; \mathrm{SD}=3.7$; range 23-35 years). All participants had hearing level not worse than $25 \mathrm{~dB} \mathrm{HL}$ at octave frequencies from $250 \mathrm{~Hz}$ to $4000 \mathrm{~Hz}$. Listeners' task was to adjust the level of music played in quiet to their most comfortable listening level and then to adjust the level of background noise to the maximum level that they still consider acceptable while listening to music. Further, music is not a speech signal that many researchers have used to determine the significance of age on ANL inter-subject variability. Results of this study supported the findings of others on age dependency, which shows that ANL inter and intrasubject variability is independent of age.
\end{abstract}

\section{Introduction}

Although, human beings are very adaptable creatures, high levels of interfering sounds are always detrimental to their effective communication. In human factors arena, these interfering sounds are commonly referred to as noise. However, the term noise has two general meanings; a narrow meaning and broader one. Noise in its narrow meaning, is a wideband sound consisting of infinite number of components with constant amplitudes and random phases (Adams \& Moore, 2009). Such noise is referred to as physical noise. One example of physical noise is thermal noise in which all components *Corresponding author. 
have similar amplitudes. Thermal noise is the noise underscoring most physical processes including spontaneous brain activity. The idealized form of thermal noise, in which all components have exactly the same amplitudes, is called white noise. Another example of physical noise is $1 / \mathrm{f}$ noise whose components have amplitudes roughly proportional to $1 / \mathrm{f}$, where $\mathrm{f}$ is the frequency of a given component. The idealized form of 1/f noise is called pink noise (Gilden, 2001). In its broad meaning, noise is any unwanted sound, including physical noise, regardless of its source.

Orthophonic (direct) speaking and listening are the primary communication modes in most educational settings. As a result, Noise Levels (NLs) and Reverberation Times (RTs) of such environments, laboratories, theater rooms, libraries, and administrative offices should be satisfactorily low that speech produced by teachers, presenters, surgeons and responding students, and audience is intelligible to all listeners (Fasanya \& Letowski, 2009). Results of Bradley (2007) study indicated that even quite modest amounts of background noise or reverberation could interfere with speech perception and consequently impair educational outcomes. Similarly, people listening to radio, TV, and other media will not understand speech and enjoy the program if the noise levels in their environment are too high. If the background noise interferes with the speech or music, the listener initially increases the signal level but eventually turns the program off if the noise level becomes unacceptably high. This situation happened often among workers performing repetitive task listens to radio to make this task less boring. Many students do read and write while at the same time listening to music in order to mask all other distracting sounds.

A number of studies have been conducted on the significance of signal-to-noise ratio (SNR) to the speech comprehension of normal listeners, as well as those who are hearing-impaired with and without hearing aids (Adams \& Moore, 2009; Gengel, 1971; Finitzo-Hieber, \& Tillman, 1978; Carhart \& Tillman, 1970; Plomp, 1976; Sutter, 1985; Dirks et al., 1982; Schum, 1996; Cooper \& Cutts, 1971; Killion, 1997). In these studies findings showed that SNR has a significant effect on speech intelligibility for both hearing impaired listeners and normal listeners. High SNR enhanced better speech intelligibility than lower SNR. About two decades ago a metric was developed to measure person's ability to function in the presence of noise; this metric is known as acceptable noise level (ANL), which is also called acceptable SNR by Nabelek et al. (1991). ANL is the maximum level of background noise listeners are willing and ready to accept without being tired or tensed when listening and following speech in a quiet condition. ANL is calculated as the difference between listener's most comfortable listening level (MCLL) and the maximum background noise level (BNL) the listener could accept (e.g. If $\mathrm{MCLL}=30 \mathrm{~dB}$ and $\mathrm{BNL}=12 \mathrm{~dB}, \mathrm{ANL}=18 \mathrm{~dB}$ ). Nabelek et al. (1991) study was to find a lasting solution to the complaint of individuals with hearing loss, and about their inability to comprehend speech when there is background noise. Several studies have been conducted on speech understanding with noise. These studies have used testing of pre and posthearing aid applications to show improved speech comprehension in noise when using hearing aids. The two goals of clinical audiologist are for patients to receive overall benefit from hearing aids and to use their hearing aids, consistently. Based on the statistics of hearing aid use, at least one of these goals is not being met (Allyson, 2006). The effort to determine factors that contribute to a person's success with amplification is perhaps one of the most challenging issues facing audiologists today.

However, several studies have shown that audiometric configuration, degree of hearing loss, type of hearing loss, embedded noise in hearing aids etc. are factors that influence people's willingness to use hearing aids (Franks, \& Beckman 1985; Garstecki, \& Erler, 1998; Kochkin, 1996; Nabelek, et al., 1991; Crowley, \& Nabelek, 1996; Allyson, 2006). The main application of ANL is to predict a person's success with hearing aids. This is also used to assess noise acceptability while listening to speech in various environmental settings. For example, Freyaldenhoven et al. (2006) showed that either acceptance of noise or the listener's willingness to wear hearing aids was significantly affected by the gain compensation of the hearing aid or the changes in the ear mold venting. In addition, the authors demonstrated a significant improvement in masked Speech Recognition Threshold (SRT) 
with binaural versus monaural amplification. It was shown that there was no improvement in ANL with binaural versus monaural amplification for most listeners. In addition, Harkrider and Smith (2005) reported that the amount of background noise, which listeners were willing to accept while listening to speech in a monotic (monaural) condition correlated with the amount of background noise they were willing to accept while listening to speech in a dichotic (binaural) condition. A study conducted by Nabelek et al. (1991) which used data from 191 listeners with hearing impairment showed that people with low ANLs $(\leq 7.5 \mathrm{~dB})$ accepted a significant amount of background noise, and were found to be successful users of hearing aids. Meanwhile, people with mid to high ANLs $(\geq 7.5$ $\mathrm{dB}$ ) accepted low background noise, and were found to be less likely to be successful users of hearing aids. This indicated that as individual's ANL increases, the possibilities of success with hearing aids decreases. Therefore, it is important to investigate the psychological and physiological processes that reduce ANL.

Moreover, researchers have consistently used speech as the signal to determine individual ANL. As an exception to all other researchers, Kattel et al. (2008) expanded on the concept of ANL metric, where they used music as a signal, believing that research has demonstrated that music defines human personality (Rentfrow \& Gosling, 2003). Their findings were similar to other researchers' findings where speech was used as the signal; ANLs for music is independent of the type of background noise used. Other researchers evaluated the effect of music as background noise and found that it has more complex influences than other types of background noise (Gordon-Hickey \& Moore, 2007). However, the study further revealed that ANL for music was likely to be better compared with ANL for twelve-talker babble. According to the authors, listeners were more willing to accept music as a background noise than speech babble. The results further revealed that ANL for the music samples were not correlated with preference for the music samples, indicating that ANL for music was not related to music preference. In 1982, Franks conducted a study on hearing aid preference judgment among normal-hearing and hearing-impaired subjects with speech and music signal. The results of the study revealed that with music signals, normal-hearing listeners pay attention to both cutoff frequencies whereas hearing-impaired listeners only took the low-frequency cutoff. Clarity was also rated high for overall music quality along with fullness, in the study conducted by Balfour and Hawkins (1992) to investigate the quality of sound stimulus. In Gordon-Hickey and Moore study, music was used as the background noise, on contrary to its usage in a study conducted by Kattel et al. (2008). Music has rarely been the subject of research in hearing science. Gordon-Hickey and Moore (2007) further documented information about researchers that have studied the use of hearing aid technology and cochlear implant technology (Gfeller et al., 2000; Leal, et al., 2003; and Kong, et al., 2004) for giving musical satisfaction to the hearing impaired.

A characteristic feature of ANL seems to be a large inter-subject variability in acceptance of background noise while listening to speech. According to Rogers et al. (2003) large inter-subject variability has been shown to be relatively independent of gender, hearing sensitivity Nabelek, et al. (2006) and Freyaldenhoven, et al. (2007) and the type of background noise distraction Nabelek, et al. (1991) and primary language of the listener Von Hapsburg and Bahng (2006). Other studies (Nabelek, et al., 1991; Freyaldenhoven \& Smiley; Nabelek, et al., 2006) have showed that ANL is independent of age. Nabelek et al. (2004) demonstrated that hearing aids type had no effect on ANLs, but speech-in-noise scores increased with the introduction of amplification. According to Nabelek (2005) acceptance of background noise is a "means" that will help to solve the puzzle and monumental problem of hearing aid rejection. Therefore, to ascertain the dependency of each factor, more than two or three authors' findings will be appropriated for a reasonable conclusion. Fasanya and Letowski (2009) substituted music for speech to determine peoples' ANL and the results proved similar to other findings. 


\subsection{Objectives of the Study}

Based on the current research findings on the subject matter, the authors hypothesized that intersubject variability in ANL will depend on age, intra-subject variability, and background noise type (signal type). Thus, the objectives of this study were to investigate the effect of age and background noise type on ANL and intra-subject variability dependency of age.

\section{Methodology}

\subsection{Participants Selection and Characteristics}

The sample included 20 undergraduate and graduate students at a public university located in the eastern region of United States. After providing them with the needed research information including the authors' commitment to their confidentiality, the students volunteered to participate in the study. The ages of the students ranged from 23 to 35, with an average age of 29 and standard deviation (SD) of 3.7. Initial screening showed that all participants had normal hearing defined as not worse than 25 $\mathrm{dB}$ HL (hearing level) at octave frequencies in the $250 \mathrm{~Hz}$ to $4000 \mathrm{~Hz}$ frequency range. Participants were diverse and consisted of ten Africans eight Afro-Americans and two Caribbean. The participants indicated their music genre preference as reggae (9), gospel (8), and hip-hop (3) for their test signal. Using Cochran (1963) sample size determination formula shown in Eq. (1), at confidence level of $90 \%$ with assumption that the population is large. Therefore, assume $p=.5$ (maximum variability), the sampling error is calculated to be $18 \%$.

$n=\frac{Z^{2} p q}{e^{2}}$

\subsection{Apparatus and Test Materials}

The study was conducted in a large Industrial Acoustics Chamber (IAC) Audiometric Booth at the Department of Industrial, Manufacturing and Information Engineering (IMIE) of Morgan State University (MSU) Baltimore, Maryland. The audiometric booth meets noise criteria for uncovered ears (American National Standards Institute, 1991) and equipped with an array of four Studiophile BX5A loudspeakers. Two of the loudspeakers were used to deliver test signals and two others were used to emit background noise. The signals ( 3 types of music) and noises ( 3 background noises) were stored on two computers with M-Audio sound cards and Sony Sound Forge 7.0 software. The three music selections used in the study were gospel recording "Leaning on the everlasting arm" by Evangelist Tolu David, hip-hop recording "Can't Hide from Love" by Jay Z and reggae recording "Tuff Gong (Is this love)" by Bob Marley \& The Wailers Legend. Each listener used the preferred music recording as the music signal. The three background noise maskers were (a) pink noise (b) white noise and (c) speech babble of 12 voices (Frank and Craig, 1984). All the signals were stored in an IBM computer and normalized to have the same relative average RMS level of $-25.0 \mathrm{~dB}$ measured at the output of the M-audio sound card. Both the music and the noise were played from IBM computers using Sony Sound Forge 7.0 software and WINAMP software for noise looping.

The positions of all volume controls except for M-audio volume control were fixed. The arrangement of the loudspeakers is shown in Fig. 1. The loudspeakers delivering background noise were positioned at the front (N2) and the back (N1) of the listener about two feet away. Two other signal loudspeakers (RS and LS) were positioned at $\pm 45^{\circ}$ azimuth and about three feet from the listener center line. The output voltage of the master volume control of the M-audio sound card was calibrated by simultaneous recording of the sound pressure levels in $\mathrm{dB}$ SPL at the right ear location of the average head height of the listener using a sound level meter. Sound pressure level was

measured using Metrosonics Chameleon sound level meter (dB SPL). Prior to the beginning of the experiment, calibration was done on sound level meter for accuracy and verified several times during 
the experiment and at its end. Listeners were instructed to maintain the same position during the experiment. The position of the listener was visible through the window of the IAC booth and the experimenter corrected the listener's position every time a noticeable departure from the initial position was noticed see (Fig. 2).

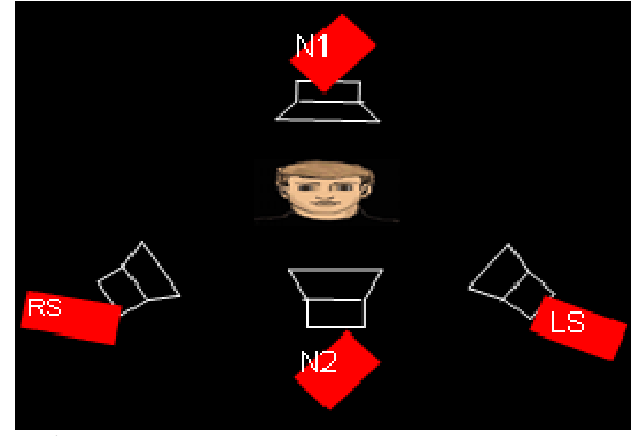

Fig. 1. Loudspeakers Arrangements in the IEC Booth (RS -Right Signal Source, LS - Left Signal Source)

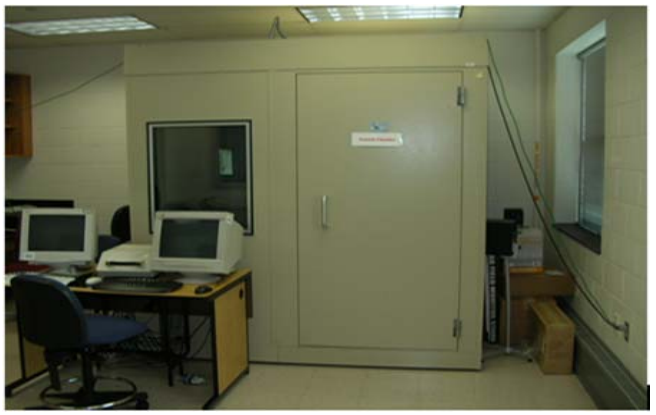

Fig. 2. Industrial Acoustics Chamber (IAC) Audiometric Booth

\subsection{Procedure}

At the beginning of the study, the experimenter explained to the each subject the purpose of the study and the subject's task. Each subject was told to imagine working in a factory doing mundane work and listening to the radio. At a certain point the level of surrounding noise increased making listening to the radio more difficult. The listener's task was to adjust the noise level to the maximum tolerable level above which he/she would stop listening or turn the radio off.

During the study, listeners were seated in the center of the IAC booth listening to sounds played by four surrounding loudspeakers (Fig. 1). There were three trials for each session. At the beginning of each experimental trial, the music was presented in quiet and adjusted to match the MCLL of the listener. Next, the signal was played at the established MCLL level for about 3 minutes after which the level of background noise was gradually increased until it reached the maximum acceptable BNL that the listener was willing to accept while listening to the signal. The ANL was calculated as the difference in $\mathrm{dB}$ between MCLL and BNL. The MCLL and BNL selected by the listener were measured as sound pressure level (dB SPL) at the point close to the right ear of the listener. Two channel listening was employed in this study. The two-channel music signals was a stereo recording in which the phantom position of the vocalist was located halfway between the RS and LS loudspeakers and aligned with the location of the front loudspeaker delivering background noise (N2). The orders of sessions and noise types within a session were randomized for each listener. Each session was conducted on different days. Each participant was tested for a little over two and halfhours.

\section{Results}

The average, range and standard deviation of ANL subject responses and the intra-subject variability results for the entire group of twenty (20) listeners based on background noise types are summarized in Table 1. This table shows that ANL mean values for white noise is higher by $6.3 \mathrm{~dB}$ than for pink noise, $7.9 \mathrm{~dB}$ for babble noise and pink noise is higher than babble noise by $1.6 \mathrm{~dB}$. The ANLs are calculated by subtracting the BNLs from the MCLLs Nabelek, et al. (1991). For example, if a listener's MCLL is $53 \mathrm{~dB}$ SPL and their BNL is $40 \mathrm{~dB}$ SPL, their ANL is $13 \mathrm{~dB}$. ANLs in this study is measured and calculated in approximately 2-3 minutes. Participants' ANL intra-subject variability results were calculated by finding the absolute values of the differences in the participant's ANL results for trial 1, trial 2 and trial 3 . The results of the absolute differences were divided by 3 . Intrasubject variability was performed so that age dependency could be viewed in two perspectives. This 
is the first time listener's ANL intra-subject variability will be used to determine the subject age dependency. Presented data were obtained using dB SPL readings of the sound level meter. Fig. 4 shows the graphical relationship between intra-subject variability and the individual ANL. It shows that at the beginning white noise and pink noise had similar structures but in average babble noise assumed closed form with white noise indicated that both would have similar effect on ANL.

How much variation exists within peoples' ANL intra-subject variability and inter-subject variability under different background noise types? As shown in Fig. 2 and Fig. 3, pictorially, it appeared that some bar showed that there is a variation within individual participants ANL for both intra and inter variability why some did not. The experimenter presumed that the variation might due to individual age differences. Therefore, an inferential statistics analysis was conducted to verify the significant of the variation to confirm (Nabelek et al., 1991; Nabelek et al., 2006; and Freyaldhoven \& Smiley in press) findings, which indicated that ANLs inter-subject variability is age independent.

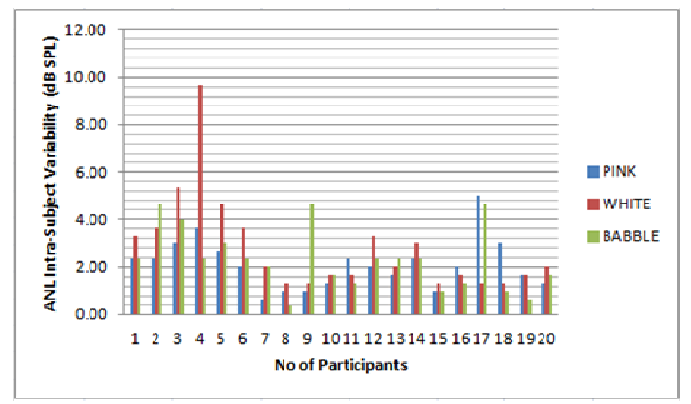

Fig. 3. Graphical Representation of ANLs IntraSubject Variability

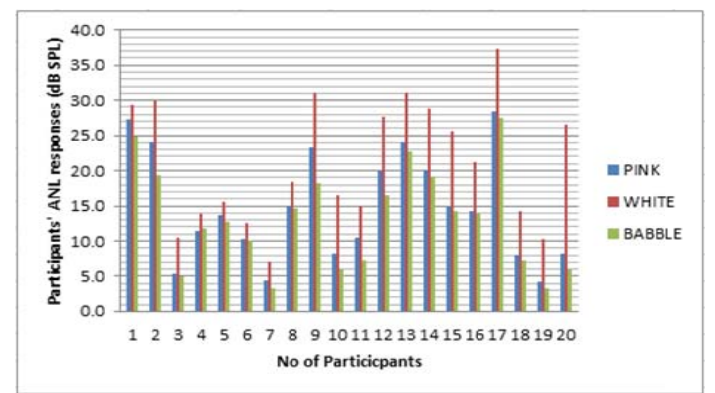

Fig. 4. Graphical Representation of ANLs InterSubject Variability

\section{Table 1}

Mean and Standard Deviation for Pink, White and Babble Noise

\begin{tabular}{lcccccc}
\hline & \multicolumn{3}{c}{ Inter-subject variability } & \multicolumn{3}{c}{ Intra-subject variability } \\
& PINK & WHITE & BABBLE & PINK & WHITE & BABBLE \\
\hline Average & 14.78 & 21.10 & 13.18 & 2.10 & 2.80 & 2.30 \\
SD & 7.75 & 8.77 & 7.23 & 1.03 & 2.01 & 1.32 \\
Range & $4.2-28.3$ & $7.0-21.1$ & $7.2-13.2$ & $0.67-5.0$ & $1.3-9.7$ & $0.33-4.67$ \\
\hline
\end{tabular}

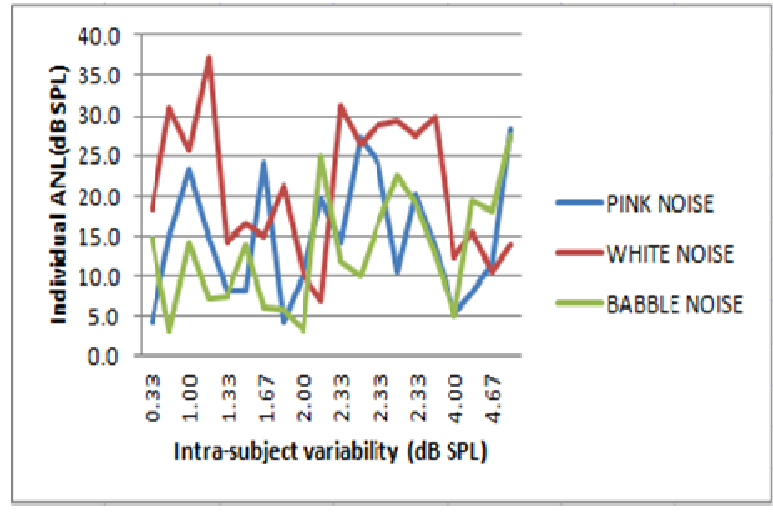

Fig. 5. Relationship between Inter and Intra-Subject Variability

SAS Inc 9.2 (2007) English version was used to analyze the data collected during this study. A multivariate analysis of variance (MANOVA) was performed to evaluate the effects of age on the ANLs inter-subject variability, and the intra-subject variability. The independent variable was age, while the dependent variables were subject ANLs and intra-subject ANL values. Results revealed that inter-subject variability under the three different types of background noise (white, pink and babble) 
were not dependent on age. Meanwhile, intra-subject ANL values for pink noise showed age dependency at $90 \%$ confident level $(\alpha=0.1)$ [F $=2.53, \mathrm{p}=0.0892]$. Overall statistics analysis showed that either inter-subject variability ANLs or intra-subject variability ANLs depends on age or background noise type with Wilks' Lambda value (0.00057) and ( $\mathrm{p}>0.05)$.

Pearson Correlation coefficients ( $\mathrm{r}$ values) were calculated to established reliability of individual responses for the twenty listeners' ANLs and the intra-subjects variability measures. The data for each of the three background noise types were compared. Correlations between ANLs values and the ANL intra-subject variability were found not to be statistically significant under different background noise types. Meanwhile ANLs intra-subject variability coefficient for babble noise was found to be significant with listeners' ANLs for both pink and babble noise, respectively $(\mathrm{p}=0.019$, and $\mathrm{p}=0.04$ and $r=0.5168$, and $r=0.46185$ ) Table 2 showed that none of the factors compared with age were statistically significant at $\alpha=0.1$. Pearson Correlation results further showed high correlation between background noise types $(r=0.9811$, between pink and white noise, $r=0.8611$ between white and babble noise and $r=0.8973$ between pink and white noise); suggesting that ANLs variability did not depend on background noise types. This is also the first time Pearson Correlation coefficient ( $\mathrm{r}$ value) will be used to determine the dependency of background noise types.

\section{Table 2}

Pearson Correlation Coefficients, for the Twenty Listeners Prob $>|r|$ under H0: Rho=0PAN-Pink Noise ANL, WAN-White Noise ANL, BAN-Babble Noise ANL, PIAN-Pink noise intra-variability ANL, WIAN-White Noise Intra-Variability ANL \& BIAN-Babble Noise Intra-Variability ANL

\begin{tabular}{rrrrrrrr}
\hline & AGE & PAN & WAN & BAN & PIAN & WIAN & BIAN \\
\hline AGE & 1.00000 & 0.21088 & 0.08936 & 0.16964 & 0.0758 & -0.20776 & 0.18587 \\
& & 0.3722 & 0.7079 & 0.4746 & 0.7508 & 0.3794 & 0.4327 \\
PAN & 0.21088 & 1.00000 & 0.89727 & 0.98121 & 0.26376 & -0.08697 & 0.51684 \\
& 0.3722 & & $<.0001$ & $<.0001$ & 0.2611 & 0.7154 & 0.0196 \\
WAN & 0.08936 & 0.89727 & 1.00000 & 0.86111 & 0.16281 & -0.25504 & 0.44286 \\
& 0.7079 & $<.0001$ & & $<.0001$ & 0.4928 & 0.2778 & 0.0505 \\
BAN & 0.16964 & 0.98121 & 0.86111 & 1.00000 & 0.33398 & -0.04093 & 0.46185 \\
& 0.4746 & $<.0001$ & $<.0001$ & & 0.1501 & 0.864 & 0.0404 \\
PIAN & 0.0758 & 0.26376 & 0.16281 & 0.33398 & 1.00000 & 0.42837 & 0.43275 \\
& 0.7508 & 0.2611 & 0.4928 & 0.1501 & & 0.0595 & 0.0567 \\
WIAN & -0.20776 & -0.08697 & -0.25504 & -0.04093 & 0.42837 & 1.00000 & 0.26128 \\
& 0.3794 & 0.7154 & 0.2778 & 0.864 & 0.0595 & 0.2658 \\
BIAN & 0.18587 & 0.51684 & 0.44286 & 0.46185 & 0.43275 & 0.26128 & 1.00000 \\
& 0.4327 & 0.0196 & 0.0505 & 0.0404 & 0.0567 & 0.2658 & \\
\hline
\end{tabular}

\section{Conclusions and Discussions}

Based on results of the analyses, ANLs obtained using pink, white and babble noise were reliable over the short period of three (3) minutes used in the experiment for listeners with normal hearing. Results were in agreement with other studies like the ones conducted by (Nabelek et al., 1991; Nabelek et al., 2006; and Freyaldhoven \& Smiley in press) which found that inter-subject variability does not depend on age, even though hearing aids users were the participants for their study. Likewise, participants' ANL inter-subject variability did not depend on background noise type. These findings confirmed some previous results (Nabelek et al., 1991). Results also showed that ANLs recorded using speech as signal can be replaced with music to determining successful use of hearing aids and for measuring human hearing fatigue. Results further showed that intra-subject variability is age independent. Interchangeability of speech to music for determining background noise and age dependency on ANL is a new finding. 
The results of this study are robust leading to conclusions on age dependency in two dimensions. It showed that any variability in ANLs in any form has nothing to do with age. Therefore, the findings of this study may be of interest to manufacturers designing hearing aids, audiologist, hearing fatigue researchers and practitioners fitting hearing aids to patients. However, some limitations were observed during this study. Time duration for participants to complete the three sessions appeared to be too much. This seemed to scare people away; thus contributing to the reduced number of participants (twenty). Future research could include hearing impaired listeners that use hearing aids in a daily basis. Background noise frequency bandwidth may also be considered in a future analysis so that concrete conclusion about background noise dependency can be drawn.

\section{Acknowledgments}

The study was not funded by any organization. However, We gratefully acknowledge the effort of Dr. Tom Letowski of Army Research Laboratory Aberdeen Proving Ground, Maryland for his significant contributions to the success of the research.

\section{References}

Adams, E. M. \& Moore, R. E. (2009). Effects of speech rate, background noise, and simulated hearing loss on speech rate judgment and speech intelligibility in young listeners. Journal of the American Academy of Audiology, 20(1), 28-39.

Allyson Adrianne Segar, Au. D. (2006). Personality type and self perception of hearing aid benefit. Dissertation, University of Maryland, College Park.

American National Standards Institute (1991). Maximum Ambient Noise Levels for Audiometric Test Rooms. (ANSI S3.1-1991). New York.

Balfour, P. B. \& Hawkins D. B. (1992). A comparison of sound quality judgments for monaural and binaural hearing aid processed stimuli. Ear and Hearing, 13, 331-339.

Bradley, J.(2007). Classroom acoustics to support student learning. In D. Jamieson, S. Rvachew, Carhart, R. \& Tillman. T.W. (1970). Interaction of competing speech signals with hearing losses. Arch Otolaryn, 91, 273-279.

Cochran, W. G. (1963). Sampling Techniques. 2nd Ed., New York: John Wiley and Sons, Inc.

Cooper, J.C. \& Cutts, B.P. (1971). Speech discrimination in noise. Speech Hearing Resources, 14, 332-337.

Crowley, H. J. \& Nabelek, A.K. (1996). Estimation of client assessed hearing aid performance based upon unaided variables. Journal of Speech Hearing Resources, 39, 19-27.

Dirks, D.D., Morgan, D.E., \&Dubno, J.R., (1982). A procedure for quantifying the effects of noise on speech recognition. Journal of Speech Hearing Disorders, 47, 114-123.

Gilden, D. L. (2001). Cognitive Emissions of 1/f Noise. The University of Texas at Austin. Austin, TX.

Fasanya, B.K. \& Letowski,T.R. (2009).Acceptable Noise Levels (ANLs) for speech and music for young people With Normal hearing. Proceedings of the HPEE Conference, San Antonio TX.

Finitzo-Hieber, T. \& Tillman, T. (1978). Room acoustics effects on monosyllabic word discrimination ability for normal \& hearing impaired children. Journal of speech Hearing Research, 21, 440-458.

Franks, J. \& Beckman N. (1985). Rejection of hearing aids: Attitudes of a geriatric sample. Ear and Hearing, 6, 161-166.

Franks, J. R. (1982). Judgments of hearing aid processed music. Ear and Hearing, 3, 18-23.

Freyaldenhoven, M. C., Plyler,P. N. Thelin, J.W. \& Burchfield, S. B. (2006). Acceptance of noise with monaural and binaural amplification. Journal of the American Academy of Audiology, 17, 659-666. 
Freyaldenhoven, M. C., Plyler, P. N., Thelin, J. W. \& Hedrick, M. S. (2007). The effects of speech presentation level on acceptance of noise in listeners with normal and impaired hearing. Journal of Speech, Language, and Hearing Research, Rockville.50 (4), 878-885.

Freyaldenhoven, M.C. \& Smiley, D.F. (in press). Acceptance of background noise in children with normal hearing. Journal of Educational Audiology.

Garstecki, D.C. \& Erler, S.F. (1998). Hearing loss, control, and demographic factors influencing hearing aid use among older adults. Journal of Speech Language, and Hearing Research, 41, 527537.

Gordon-Hickey, S. \& Moore, R.E. (2007). Influence of music and music preference on acceptable noise levels in listeners with normal hearing. Journal of American Academy Audiology, 18, 417427.

Gfeller, K., Christ, A., Knutson, J. F., Witt, S., Murray, K. T. \& Tyler, R. S. (2000).Musical backgrounds, listening habits, and aesthetic enjoyment of adult cochlear implant recipients. Journal of American Academy Audiology, 7, 390-406.

Harkrider, A. W. \& Smith, S. B. (2005). Acceptable noise level; Phoneme recognition in noise and measure of auditory efferent activity. Journal of the America academy of Audiology, 16 530-545.

Kattel, B., Fasanya, B. K. \& Letowski T. R. (2008). Acceptable Noise Level (ANL) for music listened in the background of different types of noise. Proceedings of the XX Annual International Occupational Ergonomics and Safety Conference.

Killion, M.C., (1997). SNR loss: “I can hear what people say, but I can't understand them'. Hearing Revolution Journal, 4(12), 8-14.

Kochkin, S. (1996). 10-year customer satisfaction trends in the US hearing instrument market. The Hearing Journal, 49, 23-34.

Kong, Y. Y., Cruz, R. Jones, J. A. \& Zeng F.(2004). Music perception with temporal cues in acoustic and electric hearing. Ear and Hearing, 25, 173-185.

Leal, M. C. Shin, Y. J. Laborde, M., Calmels, M., Verges, S., Lugardon, S., Deguine, O. \&Fraysse. B. (2003).Music perception in adult cochlear implant recipients. Acta Otolaryngology, 123, 826835.

Nabelek, A. K., Tucker, F.M., \& Letowski, T.R. (1991). Toleration of background noises: relationships with patterns of hearing aid use by elderly persons. Journal of Speech Hearing Resources, 34, 679-685.

Nabelek, A. K., Tampas, J. W., \&Burchfield.S. B., (2004). Comparison of speech perception in background noise with acceptance of background noise in aided and unaided conditions. Journal of Speech Hearing Resources, 47, 1001-1011.

Nabelek, A. K. (2005). Acceptance of background noise may be key to successful fittings. The Hearing Journal, 58(4), 10-15.

Nabelek, A.K., Freyaldenhoven, M.C. Tampas, J.W., Burchfield S.B. \&Muenchen, R.A. (2006). Acceptable noise level as a predictor of hearing aid use. Journal of the American Academy of Audiology, 17, 626-639.

Plomp R. (1976). Binaural and monaural speech intelligibility of connected discourse in reverberation as a function of azimuth of a single competing sound source (speech and noise). Journal of Acoustics, Society of America, 34, 200-211.

Rentfrow, P. J. \& Gosling, S. D. (2003). The Do Re Mi's of everyday life: The structure and personality correlates of music preferences. Journal of Personality and Social Psychology, 84 (6), $1236-1256$.

Rogers, D.S., Harkrider, A.W., Burchfield, S.B. \& Nabelek, A.K. (2003).The influence of listener's gender on the acceptance of background noise. Journal of the American Academy of Audiology, $14,374-385$.

Sutter, A.H. (1985).Speech recognition in noise by individuals with mild hearing impairments. Journal of Acoustics Society of America, 78(3), 887-900. 
Schum, D. (1996). Speech understanding in background noise. In M. Valente (ed.), Hearing Aids: Standards, Options, and Limitations. New York: Thieme, 368-406.

Von Hapsburg, D. \&Bahng. J. (2006). Acceptance of background noise levels in bilingual [Korean english] listeners. Journal of the American Academy of Audiology, 17, 649-658. 